
\title{
$\begin{array}{ll}\text { Research Square } & \begin{array}{l}\text { Preprints are preliminary reports that have not undergone peer review. } \\ \text { They should not be considered conclusive, used to inform clinical practice, } \\ \text { or referenced by the media as validated information. }\end{array}\end{array}$
}

\section{Acupuncture for Olfactory Dysfunction in Infected COVID-19 Patients: Study Protocol for a Single Blind, Randomized, Sham- Controlled Clinical Trial}

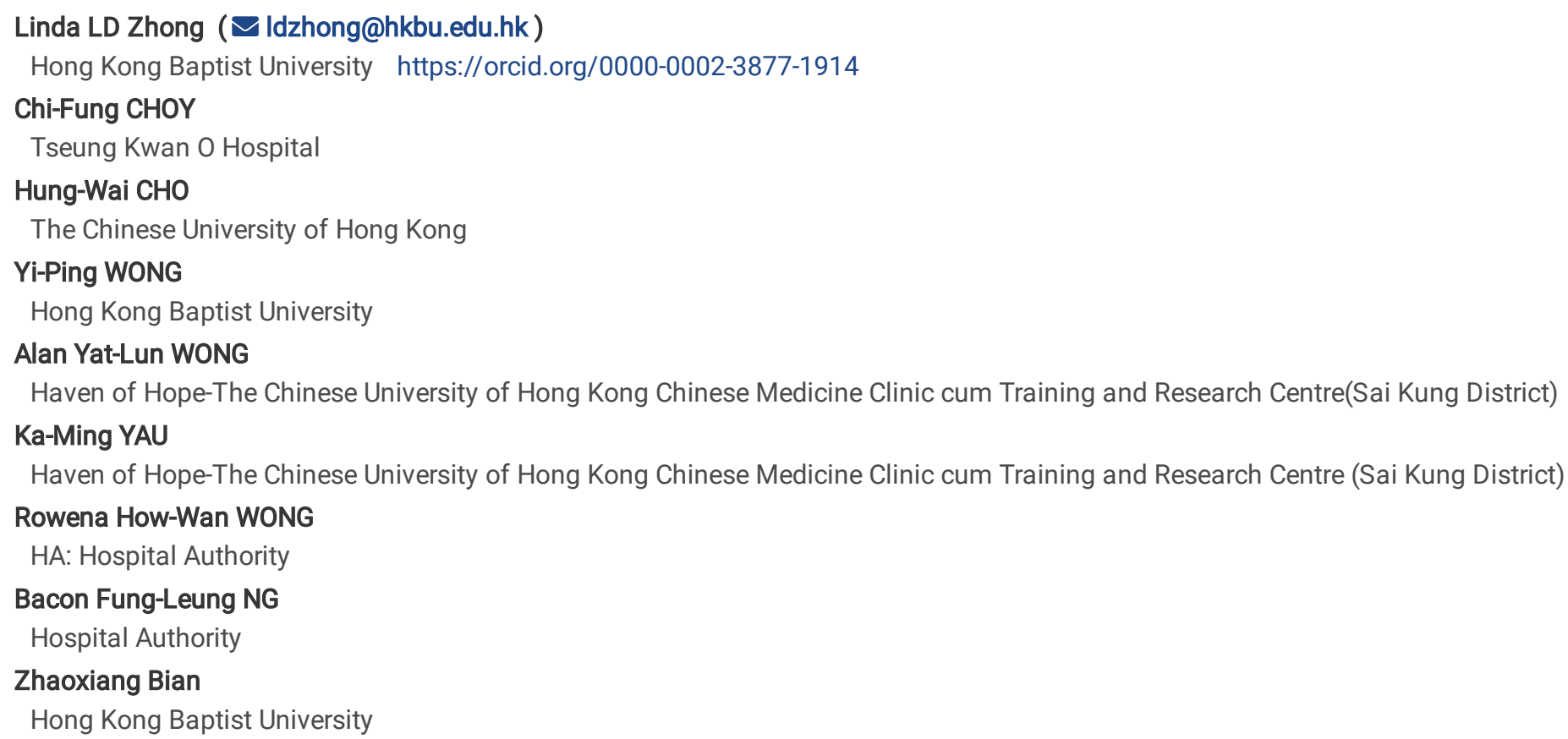




\section{Abstract}

Background: Olfactory dysfunction (OD) was recognized as one of common symptom of COVID-19. OD, defined as the reduced or distorted ability to smell during sniffing (orthonasal olfaction) and may represent one of early symptoms in the clinical course of COVID-19 infection. A large online questionnaire-based survey found that some of post COVID-19 patients showed no improvement at one month after they were discharged from hospital. Therefore, this clinical trial is designed to explore the efficacy of acupuncture for OD in infected COVID-19 patients and to determine whether acupuncture could have benefits than sham acupuncture for OD in post- COVID-19 patients.

Methods: This is a single blind, randomized controlled, cross over trial. We plan to recruit forty post-COVID-19 patients who are presenting with smell loss or smell distortions more than one month. Qualified patients will be randomly allocated into the intervention group (real acupuncture) or the control group (sham acupuncture) in a 1:1 ratio. Each patient will receive 8 sessions of treatment over 4 week (Cycle 1), and 2-week follow-up. After the follow-up, the control group will be conducted with real acupuncture for another 4 weeks (Cycle 2), and the real acupuncture group will be conducted with the 4-week sham acupuncture. The primary outcomes are the scores change on the questionnaire of olfactory functioning and olfaction related quality of life at weeks $6,8,12$ and 14 from the baseline. Secondary outcome is the change on the Olfactory Test score at the week 6 and 12 from the baseline measured by using the Traditional Chinese version of the University of Pennsylvania Smell Identification Test (UPSIT-TC).

Discussion: The results of this trial will help to determine the effectiveness of acupuncture for OD in post-COVID-19 Patients. This may provide a new treatment option for patients.

Trial registration: ClinicalTrials.gov, NCT04959747, Registered on July 13, 2021.

\section{Background}

In May 2020, olfactory dysfunction (OD) was recognized as one of a symptom of COVID-19 [1]. OD, defined as the reduced or distorted ability to smell during sniffing (orthonasal olfaction) and may represent one of early symptoms in the clinical course of COVID-19 infection. The loss of smell has been reported to occur before $(11.8 \%)$, after $(65.4 \%)$ or at the same time $(22.8 \%)$ as the onset of other general or otolaryngological symptoms [2-5].

A large online questionnaire-based survey found that, in COVID-19, OD is usually severe and sudden in onset, but transient in most patients, although $10.6 \%$ of patients showed no improvement at one month [6]. In common neurodegenerative disorders such as Alzheimer's and Parkinson's diseases have a well-known association with loss of smell. If the loss of smell is isolated, it is most likely a sensorineural cause, and of these, a substantial proportion is caused by pathogens (post-viral) [7].

In Hong Kong, more cases self-reported the sudden changes on smell loss or smell distortions which are associated with the COVID-19 infection [8]. However, due to lack of long-term follow-up, there is insufficient evidence to currently conclude to what extent this is influenced by co-existing olfactory loss or whether it is a genuine independent sequela of COVID-19. At present, evidence suggests that most patients suffer impaired gustation as a consequence of a diminished sense of olfaction and as such, the focus of assessment and treatment is directed largely towards the nose [6]. Several prognostic factors regarding the individual prognosis of untreated olfactory disorders are available. The magnitude of this impairment on the health, safety, and quality of life is a public health concern.

We therefore propose performing a patient-blind, randomized controlled, cross over study to evaluate whether acupuncture may be beneficial to psot-COVID-19 patients who have moderate or severe grades OD.

\section{Objectives}

Through this study, we expect to assess the effects of acupuncture for Olfactory Dysfunction patients who did not recover olfaction after infected COVID-19 and to compare the results with the impact observed in a sham acupuncture.

\section{Methods}

\section{Study design}

This is a single blind, randomized sham controlled, and cross-over clinical trial. Post- COVID-19 patients who presenting to smell loss or smell distortions will be recruited from the Department of Medicine to the Department of Ear, Nose and Throat (ENT) in Tseung Kwan 0 Hospital for further assessment. Screening will be conducted in Department of ENT on Visit 0, using the Traditional Chinese version of the 
University of Pennsylvania Smell Identification Test (UPSIT-TC). Patients who with moderate or severe grades (base on UPSIT-TC scores, the cut-off scores are set at 29.5 for male and 30.5 for female) will be recruited. Forty qualified patients will be invited to participate in the study and be referred to the CMCTR for the further action, such as written informed consent, assessment, acupuncture treatment etc.

The objective of this study is to investigate the OD of COVID-19 patients with subjective validated patient-reported outcome questionnaires and objective psychophysical testing.

The protocol has been approved by Hong Kong Baptist University Human Research Ethics(Clinical) Committee on the Use of Human Subjects for Teaching and Research (Approval no. REC/20-21/0508), Hospital Authority Kowloon Central/ Kowloon East Research Ethics Committee (Approval no. KC/KE-21-0054/FR-3) and registered in clinicaltrials.gov (NCT04959747). The Study flow chart listed in Fig. 1. Trials (SPIRIT) checklist is provided as Additional file 1 and the schedule of enrolment, intervention, and assessments is shown in Fig. 2 (SPIRIT figure)

\section{Setting and Recruitment}

The study will be conducted in three Department at Tseung Kwan O Hospital (TKOH), Hong Kong. Department of Medicine, Department of ENT and Department of Chinese Medicine: Haven of Hope - The Chinese University of Hong Kong Chinese Medicine Clinic cum Training and Research Centre (CMCTR).

\section{Inclusion criteria}

Patients who have been previously diagnosed to be infected with COVID-19 and are discharged from local hospitals with all the follows: 1) Post COVID-19 olfactory dysfunction with moderate or severe grades (base on UPSIT-TC scores, the cutoff scores are set at 29.5 for male and 30.5 for female); 2) Post COVID-19 olfactory dysfunction who did not undergo treatment; 3 ) No history of trauma, injury or surgery to head or nose or any bleeding from the nose; 4) Aged 18 to 80 years who are able to read and write Chinese.

\section{Exclusion criteria}

Patients will be excluded if they have one or more of follows: 1) patients with olfactory or gustatory dysfunctions before the COVID-19 epidemic; 2) patients with history of chronic rhinosinusitis or nasal polyposis; 3 ) history of nasal surgery (including rhino/septoplasty with or without functional endoscopic sinus surgery; 4) pregnant or breastfeeding women.; 5) Medical history: cancers, conditions that affect the nervous system, such as Parkinson's disease or Alzheimer's disease, and/or any other serious diseases; 6) Unstable medical conditions; 7) Patients who have received acupuncture treatment within one month, 8) Alcoholism or drug abuse in past 1 year; 9) Have needle phobia; 10) Have known history of developing acupuncture related severe adverse reaction.

\section{Screening}

Post COVID-19 patients presenting with smell loss or smell distortions will be referred from the Department of Medicine to the Department of ENT in TKOH for further assessment. Screening will be conducted in Department of ENT of TKOH on Visit 0, using UPSIT-TC (a 40-item olfactory test which is widely used internationally). Forty qualified patients will then be invited to participate in the study and be referred to the CMCTR for the further action.

\section{Baseline visit}

After screening, all eligible patients will be scheduled a baseline visit in the CMCTR. During the visit, the patient will be further assessed by the Chinese Medicine Practitioner (CMP) CMP will using the 12-item validated questionnaire for the assessment of self-reported olfactory functioning and olfaction related quality of life (ASOF) and the short version of the Questionnaire of Olfactory Disorders-Negative Statements (SQOD-NS). The answers to the ASOF and SQOD-NS reported by the patient are to be filled in by the study team.

The CMP will inform the patient the details of the study and obtain his/her written informed consent.

Upon signing the informed consent, the patient will then be randomly assigned into either the acupuncture group (AC) or the sham acupuncture group (SAC), Intervention (Cycle 1 treatment period) will start after a 2-week run in period.

\section{Randomization and blinding}

Forty qualified patients will be randomly assigned into 2 groups, the acupuncture group (AC) and the sham acupuncture group (SAC) with 1:1 ratio, for 4-week treatment and 2-week follow-up. After the follow-up, the sham group will be conducted with real acupuncture for another 4 weeks and the real acupuncture group will be conducted with the 4-week sham acupuncture. 
Simple, complete, non-sequential randomization numbers will be generated by Random Allocation Software (Version 2.0.0), Isfahan, Iran and kept by Principal Investigator (PI). To reduce the risk of breaking the blind, hence reducing bias as much as possible, the trial is patientblind, data collection staff and data analysts also are blinded during the study period. The acupuncturist is not blinded to the treatment to be delivered because acupuncture manipulation makes this impossible.

The randomization list is only available to the $\mathrm{PI}$ and the acupuncturist, and will not be available to any person involved in the conduct and evaluation of the trial until the trial database is locked.

\section{Sample size and calculation}

Since it is a pilot study and there is no acupuncture study for COVID-19 patients with OD. Sample size calculation are based on the primary outcome measurement. We assume that there will be $30 \%$ improvement of acupuncture group compared with control group. Considering the power of $80 \%$, and alpha value of $2.5 \%$ (two-tailed), each group will need at least thirty-two subjects to detect for the significance to be detected. Allowing for a $20 \%$ drop-out, we plan to recruit forty subjects in each group. As this is a cross-over design, so the total sample size is eighty and we only need to recruit forty patients.

\section{Intervention}

Each patient will be scheduled for 8 sessions of acupuncture treatment (Cycle 1), 30 minutes for each session, twice per week over a 4week period. Selection of acupuncture points is based on Traditional Chinese Medicine (TCM) theory $[12,13]$ and evidence-based clinical research [14] in the CMCTR. According to the TCM theory, Yin Tang is a local point and is indicated in the treatment of frontal headache, eye disorders, rhinitis, sinusitis and insomnia. LI20 in Chinese is Yingxiang which means 'welcome fragrance'. LI20 is indicated in nasal obstruction and loss of sense of smell. Needling LI20, in traditional terms, helps the 'lung' to perform its dispersing function, so that the nose can smell fragrant scent, hence the name 'welcome fragrance' and it for the treatment of anosmia. GV23 (Shangxing) was used as a local point for the nose and over frontal sinus [15-19]. Thus, the acupuncture points selected for treatment are listed in Table 1 and Fig. 3 (Total of 8 needles for 8 acupuncture points on each acupuncture treatment session).

\section{Education of acupuncturists}

The acupuncturists assigned to perform acupuncture intervention on the participants are CMPs at the CMCTR, who have received training for acupuncture and have at least three years of clinical experience in performing acupuncture and with experience in treating OD.

Table 1

Acupuncture Points

\begin{tabular}{|lll|}
\hline $\begin{array}{l}\text { Acupuncture } \\
\text { point }\end{array}$ & Anatomical location & Function according to TCM \\
\hline $\begin{array}{l}\text { Yingxiang } \\
\text { LI20) }\end{array}$ & $\begin{array}{l}\text { In the nasolabial groove, at the level of the } \\
\text { midpoint of the lateral border of ala nasi }\end{array}$ & Dispels exterior wind. \\
\hline BiTong & $\begin{array}{l}\text { On the region of the face, at the junction of } \\
\text { the cartilage of the ala nasi and the nasal } \\
\text { concha, near the upper end of the } \\
\text { nasolabial groove. }\end{array}$ & Benefits the nose. \\
\hline $\begin{array}{l}\text { Shangxing } \\
\text { (GV23) }\end{array}$ & $\begin{array}{l}\text { 1 cun directly above the midpoint of the } \\
\text { anterior hairline. }\end{array}$ & Opens the nose \\
\hline Yintang & $\begin{array}{l}\text { Midway between the medial ends of the two } \\
\text { eyebrows. }\end{array}$ & $\begin{array}{l}\text { Eliminates wind, stops convulsions and calms the mind. } \\
\text { Hegu (LI4) }\end{array}$ \\
\hline $\begin{array}{l}\text { On the dorsum of the hand, between the 1st } \\
\text { and 2nd metacarpal bones, approximately } \\
\text { in the middle of the 2nd metacarpal bone on } \\
\text { the radial side. }\end{array}$ & $\begin{array}{l}\text { Dispels exterior wind, releases the exterior, stimulates the dispersing } \\
\text { function of the Lungs, stops pain, removes obstructions from the } \\
\text { channel, tonifies Qi and consolidates the exterior, harmonizes } \\
\text { ascending and descending functions. }\end{array}$ \\
\hline
\end{tabular}

\section{Acupuncture (AC) group}

Disposable acupuncture needles provided by CMCTR $(0.25 \mathrm{~mm}$ in diameter and 25-30mm in length) are inserted at a depth of $10-25 \mathrm{~mm}$ obliquely into scalp acupuncture points (ShangXing, YinTang) and straightly into face/body acupuncture points (Yingxiang, BiTong, Hegu). Electro-acupuncture will be applied to the face points at fast and dispersed waves through electric needle stimulator which is provided by 
CMCTR (ES-160 6-Channel Programmable Electro-acupuncture) for $30 \mathrm{~min}$. The intensity is adjusted to a level at which patients feel comfortable. The alternating stimulation is believed to produce maximal biochemical responses in the brain [20].

\section{Sham-Acupuncture (SAC) group}

For subjects assigned to control group, School of Chinese Medicine, Hong Kong Baptist University will provide Streitberger's non-invasive acupuncture needles. It will be applied to serve as sham control at the same acupoints with the same stimulation modality, except that the needles are only adhered to the skin instead of insertion. Its validity and credibility have been well demonstrated.

The placebo needle (SAC group) looks like a real acupuncture needle and appears to penetrate the skin like a real acupuncture needle. It will be applied at the real acupuncture points with the same stimulation modality except that the needles are only adhered to the skin by a small plastic ring instead of being inserted and the stimulation will be a "pseudo stimulation", which will be given by connecting the needle to an incorrect output socket of the electrical acupuncture stimulation instrument [9]. Therefore, the patients cannot visually detect sham or active acupuncture procedure.

The participants will not be able to tell the difference of a sham acupuncture procedure from the active acupuncture procedure, because as the placebo needle was pushed against the skin, it caused a pricking sensation. However, as increased pressure is applied, the shaft of the placebo needle disappears into the handle, which gives the impression that the placebo needle is actually entering the skin [10]. In Streitberger's research, it was reported that none of the 60 volunteers suspected that the needle might not have penetrated the skin [11].

\section{Outcome Measures}

\section{Assessments and time-points}

Assessments will be performed at baseline and every protocol-defined interval (Table 2) until the study completion or the study drop out. 
Schedule of study visits

\begin{tabular}{|c|c|c|c|c|c|c|c|c|c|c|c|c|c|c|c|c|c|c|c|}
\hline \multirow{2}{*}{$\begin{array}{l}\text { Assessments } \\
\text { and } \\
\text { procedures } \\
\text { Visit }\end{array}$} & \multirow{2}{*}{$\begin{array}{l}\text { Screening } \\
0\end{array}$} & \multirow{2}{*}{$\begin{array}{l}\text { Baseline } \\
1\end{array}$} & \multicolumn{8}{|c|}{ Cycle 1 Treatment period } & \multicolumn{8}{|c|}{ Cycle 2 Treatment period } & \multirow{2}{*}{$\begin{array}{l}\text { Last } \\
\text { Follow- } \\
\text { up } \\
18\end{array}$} \\
\hline & & & 2 & 3 & 4 & 5 & 6 & 7 & 8 & 9 & 10 & 11 & 12 & 13 & 14 & 15 & 16 & 17 & \\
\hline $\begin{array}{l}\text { Check and } \\
\text { confirm } \\
\text { participant } \\
\text { eligibility }\end{array}$ & $x$ & & & & & & & & & & & & & & & & & & \\
\hline UPSIT-TC & $\mathrm{x}$ & & & & & & & & & $x$ & & & & & & & & $x$ & \\
\hline ASOF & & $x$ & & & & & & & & $x$ & $x$ & & & & & & & $x$ & $x$ \\
\hline sQOD-NS & & $x$ & & & & & & & & $x$ & $x$ & & & & & & & $x$ & $x$ \\
\hline $\begin{array}{l}\text { Acupuncture } \\
\text { session }\end{array}$ & & & $x$ & $x$ & $x$ & $x$ & $x$ & $x$ & $x$ & $x$ & $x$ & $x$ & $x$ & $x$ & $x$ & $x$ & $x$ & $x$ & \\
\hline $\begin{array}{l}\text { 2-week } \\
\text { Follow up } \\
\text { visit after } \\
\text { cycle } 1 \\
\text { treatment }\end{array}$ & & & & & & & & & & & $x$ & & & & & & & & \\
\hline $\begin{array}{l}\text { 2-week } \\
\text { Follow up } \\
\text { visit after } \\
\text { cycle 2 } \\
\text { treatment } \\
\text { (last follow } \\
\text { up) }\end{array}$ & & & & & & & & & & & & & & & & & & & $x$ \\
\hline \multicolumn{20}{|c|}{ Remarks: There will be 19 study visits in total. } \\
\hline \multicolumn{20}{|c|}{$\begin{array}{l}\text { UPSIT-TC: to be conducted in Dept of ENT, TKOH in Visit } 0,9 \text { and Visit 17. For Visit } 9 \text { and } 17 \text {, the UPSIT-TC in Dept of ENT will be } \\
\text { scheduled on the same day as the acupuncture session, ASOF and sQOD-NS in the CMCTR. }\end{array}$} \\
\hline upuncture s & n: to be & ucted & $C M$ & & the & $M C$ & $\mathrm{D}$ in & Yinit & & & & & & & & & & & \\
\hline
\end{tabular}

\section{Primary outcomes}

The primary outcomes are the assessment of self-reported olfactory functioning and olfaction related quality of life (ASOF) and short version of Questionnaire of Olfactory Disorders-Negative Statements (sQOD-NS).

The ASOF is a 12-item validated questionnaire, it can be subdivided into three domains: the one-item subjective olfactory capability scale (SOC), the five-item self-reported capability of perceiving specific odors scale (SRP), and the six-item olfactory-related quality of life (ORQ) scale. We proceeded to calculate cutoff scores for the three scales. Patients will be considered to have abnormal olfactory capabilities if the SOC score was equal to or less than 3. Patients were considered to have problems smelling odors if the SRP score was equal to or less than 2.9. Patients were considered to have smell-related problems in quality of life if the ORQ score was equal to or less than 3.7 [21].

The SQOD-NS is a 7-item patient-reported outcome questionnaire. Patients rated the item proposition from 0 (agree) to 3 (disagree) with a total score ranging from 0 (significant impact of olfactory dysfunction on QOD) to 21 (no impact on QOD). The sQOD-NS is composed of seven questions including social, eating, annoyance, and anxiety and assessed using a 4-point Likert scale (0-3). A higher score means better olfactory specific QOD [22].

\section{Secondary outcome}

Secondary outcome is the Traditional Chinese version of the University of Pennsylvania Smell Identification Test (UPSIT-TC).

The University of Pennsylvania Smell Identification Test (UPSIT) is widely used internationally and the worldwide standard for olfactory testing. UPSIT is a 40-item "scratch and sniff" multiple choice test to assess how well participants can identify different odors. Patients will be required to identify each of the 40 odors from four alternatives listed for each odor. The number of correct responses provided a test 
score such that patients could be categorized into one of four categories: mild, moderate, severe, or total loss. The test is scored by the number of odors identified correctly to generate a maximum score of 40 .

UPSIT-TC is a modified version of UPSIT in Traditional Chinese developed for administration in Taiwan, with the deletion of a few odors that are unfamiliar and addition of a few odors that are familiar to the Taiwanese populations.

Due to the lack of validated study in Hong Kong and concrete data for Hong Kong population, the UPSIT-TC and its validated cutoff scores used in Taiwan are currently being used in the clinical practice in Hong Kong.

The UPSIT-TC cutoff scores for an olfactory diagnosis of our study are set based on the validated study in Taiwan, the 'Establishment of olfactory diagnosis for traditional Chinese version of University of Pennsylvania Smell Identification Test, 2016' [23], which based on the assessment of the 1440 enrolled subjects, an olfactory diagnosis of UPSIT-TC has been established in relation to gender and age, and the cutoff scores between normosmia and mild hyposmia were established at 29.5 for males aged 20 to 59 years, and the cutoff scores were established at 30.5 for females aged 20 to 59 years $[23,24]$.

The different cutoff scores between male and female adults were further analyzed in another study published in 2019, the 'Sex Differences in Human Olfaction: A Meta-Analysis', the results of their meta-analysis indicated that women generally outperform men in olfactory abilities [25].

\section{Data Collection, Management And Analysis}

An independent data monitoring committee will be established for monitoring the quality, accuracy, and completeness of all data collected periodically. Training will also be provided to all study personnel and cross-validation through interrater reliability will be performed with Universities' expert involvement.

Data monitoring committee will be established and led by the Principal Investigators (PIs). Co-investigator (Co-l) are responsible for monitoring the quality, accuracy, and completeness of all data collected periodically. Training will also be provided to all study personnel and cross-validation through interrater reliability will be performed with Universities' expert involvement.

Data management

A senior research assistant (RA) will be responsible for handling the data to ensure the authenticity and accuracy of study data. All electronic data will be password protected. Files are anonymous and assigned with a unique subject code. The completed questionnaires (hard copies) will be kept in a locked cabinet inside study sites or research offices. Study data is kept anonymous with unidentifiable code on study documents and will follow the HK Hospital's policy on handling, storage, and destruction of patient data privacy.

\section{Statistical analysis}

All efficacy and safety analyses will be conducted according to the intention-to-treat (ITT) principle. Missing values will be imputed by the last-observation-carried forward method. The statistical analysis will be performed using the Statistical Packages of Social Sciences (SPSS) for Windows version 25.0. The statistical significance is defined as two-sided P-value of $<0.05$. Baseline differences between the groups will be assessed with the use of Student's t-test for normally distributed continuous variables and nonparametric Mann-Whitney $U$ test for non-normally distributed. For categorical variables, chi-squared test or Fisher's exact test will be used. Changes in scores at each evaluation time point from baseline will be analyzed using ANCOVA model, followed by Bonferroni t-test to detect differences between two groups at each time point. The same analysis measure will be repeated within the two different treatment cycles of each subject to examine the within-subject difference. Incidence of adverse events will be examined using Chi-square test.

\section{Plans to promote participant retention and complete follow-up}

This study requires the eligible participants to attend assessment sessions and follow-up visit, and to fill in questionnaires and undergo related assessments. To maximize the participants' compliance, a thorough consent process will be provided for all participants, with details of the study schedule, the participants' responsibilities, and our support. Participants are free to withdraw at their discretion without penalty. The research team will attempt to call the participants who dropped out.

\section{Dissemination plans}


RA will be responsible for handling the data to ensure the authenticity and accuracy of study data. Only PIs, Co-I, RA, and regulatory authorities (e.g. monitors, auditors, ethic committees) have authority to check the documents.

\section{Confidentiality}

The personal data will be used for research purposes only. All the data will be destroyed after 3 years of the completion of the research study.

\section{Potential risks and patient safety}

Acupuncture is a safe method of treatment, but it may have side effects including: bruising, minor bleeding, numbness or tingling at or near the needle site, which may last a few days. Unusual risks of acupuncture include fainting, spontaneous miscarriage, nerve damage, and organ puncture. Infection is another possible risk, however since we only use sterilized, disposable needles while maintaining a clean and safe environment, this is unlikely.

Moreover, if patients feel unwell during the acupuncture treatment, we will be stopped immediately, and will be treated as soon as possible. The study is also covered by professional liability insurance policy.

\section{Discussion}

The sense of smell is great importance in our daily life. It continuously informs us about our surroundings, such as warns us about potential air hazards, and along with the sense of taste, it determines the taste and smell of food etc. And it is a strong connection between smells and memory, with olfaction having a great influence on a person's emotional state. Thus, the magnitude of olfaction impairment on the health, safety, and quality of life is a public health concern.

In Hong Kong, more cases self-reported the sudden changes in smell loss or smell distortions which are associated with the COVID-19 infection. However, due to lack of long-term follow-up, there is insufficient evidence to currently conclude to what extent this is influenced by co-existing olfactory loss or whether it is a genuine independent sequelae of COVID-19.

However, our study has a limitations. First, the sample size of the trail should be bigger Due to the pandemic is reducing in Hong Kong recently, it may be difficult to recruit patients shortly. Future studies need to increase the sample size and determine whether the observed increase of olfactory sensitivity is temporary or is lasting for a longer period of time.

Acupuncture played an active role in traditional Chinese medicine. The result of this trial is expected to provide convincing evidence that acupuncture is effective for OD in post-COVID-19 patients and whether traditional acupuncture theories play an important role in it.

\section{Abbreviations}

AC

acupuncture group

ASOF

Assessment of self-reported olfactory functioning and olfaction related quality of life

CMCTR

Chinese Medicine Clinic cum Training and Research Centre

CMP

Chinese Medicine Practitioner

COVID-19

coronavirus disease 2019

ENT

Ear, Nose and Throat

HA

Hospital Authority

OD

Olfactory dysfunction

ORQ

olfactory-related quality of life

SAC 
Sham acupuncture group

SRP

Self-reported capability of perceiving specific odors scale

sQOD-NS

Short version of the Questionnaire of Olfactory Disorders-Negative Statements

TKO

Tseung Kwan O Hospital

UPSIT-TC

Traditional Chinese version of the University of Pennsylvania Smell Identification Test

\section{Declarations}

\section{Trial status}

The protocol version number is 1.0 dated 15042021 . This study not actively recruiting.

\section{Acknowledgements}

We would like to thank Sonia Goh, Research Assistant, revision of the protocol and CRF. Written informed consent was obtained from the patient(s) for publication of this manuscript and accompanying images. A copy of the written consent is available for review by the Editorin-Chief of this journal.

\section{Authors' contributions}

LDZ conceived of the study. YPW drafted the manuscript. LDZ, CFC, HWC, HHW and FLN initiated the study design. CFC, HWC and YLW helped with its implementation. LDZ, CFC, HWC critically revised the manuscript for important intellectual content.All authors made a substantial contribution to the refinement of the study protocol and approved the final version of this manuscript.

\section{Funding}

This research was financially supported by Hong Kong Hospital Authority (Ref Code: HA105/48 PT35). The funders have no role in the trial design, the intervention procedures, collection, evaluation, and analysis of data.

\section{Availability of data and materials}

The corresponding author has access to the final trial information, and the data will be available on reasonable request (Linda ZHONG, Contact: Idzhong@hkbu.edu.hk)

\section{Ethics approval and consent to participate}

The protocol has been approved by Hong Kong Baptist University, Human Research Ethics(Clinical) Committee on the Use of Human Subjects for Teaching and Research (Approval no. REC/20-21/0508), Hospital Authority Kowloon Central/ Kowloon East Research Ethics Committee (Approval no. KC/KE-21-0054/FR-3) and registered in clinicaltrials.gov (NCT04959747). The investigators declare the trial has received ethical approval from the appropriate ethical committee, as described above.

Written consent will be taken from all the eligible and willing participants before their participation in the study.

\section{Consent for publication}

Not applicable.

\section{Competing interests}

The authors declare that they have no competing interests.

\section{Authors' information}

Affiliations 
School of Chinese Medicine, Hong Kong Baptist University, 3/F, Jockey Club School of Chinese Medicine Building, 7 Baptist University Road, Kowloon Tong, Kowloon, Hong Kong.

Linda L. D. Zhong: Idzhong@hkbu.edu.hk;_Yi-Ping WONG:ypwong@hkbu.edu.hk;

Zhaoxiang Bian: bzxiang@hkbu.edu.hk

Department of Medicine, Tseung Kwan O Hospital, Hospital Authority, Hong Kong.

Chi-Fung CHOY:ccf011@ha.org.hk

Department of Ear, Nose and Throat (ENT) of Tseung Kwan 0 Hospital, Hong Kong.

Hung-Wai CHO: chw553@ha.org.hk

Haven of Hope - The Chinese University of Hong Kong Chinese Medicine Clinic cum Training and Research Centre (Sai Kung District), Hong Kong.

Alan Yat-Lun WONG:alanylwong@hohcs.org.hk ; Ka-Ming YAU: samyau@hohcs.org.hk

Chinese Medicine Department, Hong Kong Hospital Authority, Kowloon, Hong Kong.

Rowena How-Wan WONG: rowena.wong@ha.org.hk, Bacon Fung-Leung NG: ngfl@ha.org.hk;

\section{References}

1. UK Department of Health and Social Care. Statement from the UK Chief Medical Officers on an update to coronavirus symptoms: 18 May 2020. https://www.gov.uk/government/news/statement-from-the-uk-chief-medical-officers-on-an-update-to-coronavirussymptoms-18-may-2020.

2. Lechien JR, Chiesa-Estomba CM, De Siati DR, et al. Olfactory and gustatory dysfunctions as a clinical presentation of mild-to-moderate forms of the coronavirus disease (COVID-19): a multicenter European study. Eur Arch Otorhinolaryngol. 2020. doi:10.1007/s00405-02005965-1.

3. Jay F, Piccirillo MD. Washington University School of Medicine, Coronavirus Smell Therapy for Anosmia Recovery (Co-STAR). https://clinicaltrials.gov/ct2/show/NCT04422275.

4. The Prevalence of Olfactory and Gustatory Dysfunction in COVID-19 Patients: A Systematic Review and Meta-analysis. 2020 Jul; 163(1):3-11. https://pubmed.ncbi.nlm.nih.gov/32369429/"ubMed.

5. Centers for Disease Control and Prevention. Coronavirus disease 2019 (COVID-19)-symptoms. Published April 17, 2020.

6. Walker A, Pottinger G, Scott A, Hopkins C. Anosmia and loss of smell in the era of COVID-19, BMJ 2020; 370, doi: https://doi.org/10.1136/bmj.m2808.

7. Self-reported alteration. of sense of smell or taste in patients with COVID-19: a systematic review and meta-analysis on 3563 patients, Rhinology 2020. doi:10.4193/Rhin20.185. [PubMed].

8. Chung TWai-Hin, Sridhar S, Zhang AJ. Olfactory Dysfunction in Coronavirus Disease 2019 Patients: Observational Cohort Study and Systematic Review. Open Forum Infectious Diseases. June 2020;7, Issue 6:ofaa199. https://doi.org/10.1093/ofid/ofaa199.

9. Chan K, Lui L, Yu K, Lau K, Lai M, Lau W, et al. The efficacy and safety of electro-acupuncture for alleviating chemotherapy-induced peripheral neuropathy in patients with colorectal cancer: study protocol for a single-blinded, randomized sham-controlled trial. Trials. $2020 ; 21$.

10. Xie C-c, Wen X-y, Jiang L, Xie M-j, Fu WB. Validity of the "Streitberger" Needle in a Chinese Population with Acupuncture: A Randomized, Single-Blinded, and Crossover Pilot Study. Evidence-Based Complementary and Alternative Medicine. 2013; $2013: 251603$.

11. Streitberger K, Kleinhenz J. Introducing a placebo needle into acupuncture research. Lancet. 1998 Aug 1; 352(9125):364-5. doi: 10.1016/S0140-6736(97)10471-8. PMID: 9717924.

12. Yuan HU, Lei SHI, Shouji ZHANG. TCM treatment of olfactory disorders after virus infection, Chin J Otorhinolaryngol Integ Med 2018.Vol.26.No.6, doi:10.16542/j.cnki.issn.1007-4856.2018.06.007.

13. China Academy of Chinese Medical Sciences. Evidence-based Guidelines of Clinical Practice in Chinese Medicine Internal Medicine. China Press of Traditional Chinese Medicine; 2011. 
14. Shen XY, Wang H, editors. Acupuncture and Moxibustion. People's Medical Publishing House; 2007.

15. Hecker HU, Steveling A, Peuker E, Kastner J, Liebchen K. Color Atlas of Acupuncture. Stuttgart: Georg Thieme Verlag; 2001.

16. Li D. Acupuncture. Meridian Theory and Acupuncture Points. Berkeley: Pacific View Press; 1992.

17. So JTY. The Book of Acupuncture Points. Brookline: Paradigm Publications; 1985.

18. De Smul A, Anosmia. Hyposmia Treatment with Electro-acupuncture. Acupunct Electrother Res. 1987;12(3-4):260.

19. Yang J, Zhang Q. Twenty-three cases of atrophic rhinitis treated by deep puncture at three points in the nasal region. J Tradit Chin Med. 1999;19(2):115-7.

20. Han JS. Acupuncture: neuropeptide release produced by electrical stimulation of different frequencies. Trends Neurosci. 2003;26:1722.

21. Hufnagl B, Lehrner J, Deecke L. Development of a questionnaire for the assessment of self-reported olfactory functioning. Chem Senses. 2003;28:E27.

22. Mattos JL, Edwards C, Schlosser RJ, Hyer M, Mace JC, Smith TL, Soler ZM. A brief version of the questionnaire of olfactory disorders in patients with chronic rhinosinusitis. Int Forum Allergy Rhinol. 2019;9(10):1144-50. doi:10.1002/alr.2239.

23. Jiang RS, Liang K. Establishment of olfactory diagnosis for traditional Chinese version of University of Pennsylvania Smell Identification Test. Int Forum Allergy Rhinol. 2016;6(12):1308-14.

24. Jiang Po-YuLRong-San. Prognosis of olfactory and gustatory dysfunctions in COVID-19 patients: A case series. Clin Case Rep. 2020;8:2744-52. DOI:10.1002/ccr3.3269.

25. Sorokowski P, Karwowski M, Misiak M, Marczak MK, Dziekan M, Hummel T, et al. Sex Differences in Human Olfaction: A Meta-Analysis. Front Psychol. 2019;10:242. https://doi.org/10.3389/fpsyg.2019.00242.

\section{Figures}

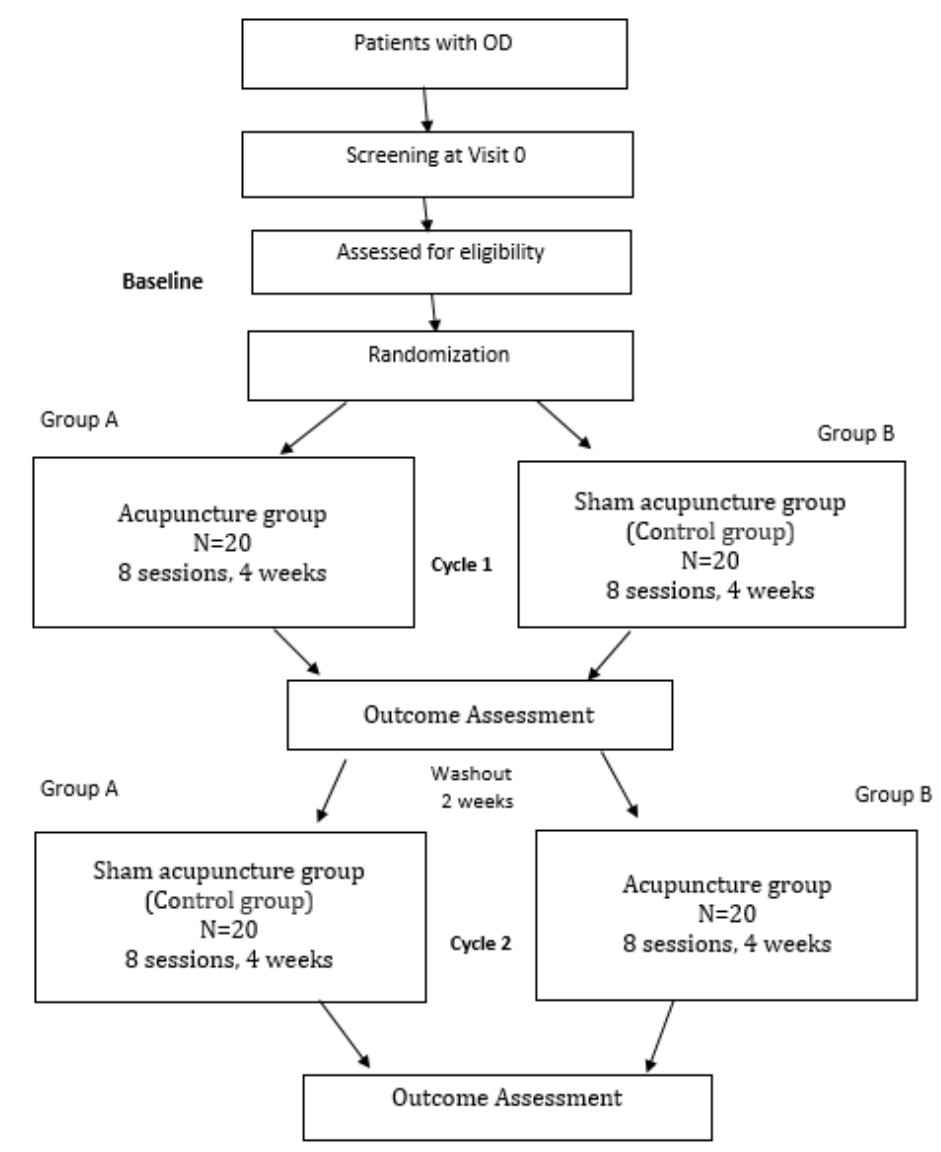

Figure 1

Study flow chart 


\begin{tabular}{|c|c|c|c|c|c|c|c|c|c|}
\hline \multirow[b]{4}{*}{ TIMEPOINT } & \multirow[b]{4}{*}{ Visit } & \multicolumn{8}{|c|}{ STUDY PERIOD } \\
\hline & & \multirow{3}{*}{$\begin{array}{c}\text { Enrolment } \\
0\end{array}$} & \multirow{3}{*}{$\begin{array}{c}\text { Baseline } \\
1\end{array}$} & \multicolumn{5}{|c|}{ Treatment } & \multirow{3}{*}{$\begin{array}{c}\text { Follow-up } \\
18\end{array}$} \\
\hline & & & & \multicolumn{2}{|c|}{ Cycle 1} & \multicolumn{3}{|c|}{ Cycle 2} & \\
\hline & & & & $2-8$ & 9 & 10 & $11-16$ & 17 & \\
\hline \multicolumn{10}{|c|}{ ENROLMENT: } \\
\hline \multicolumn{2}{|c|}{ Eligibility screen } & $\mathrm{x}$ & & & & & & & \\
\hline \multicolumn{2}{|c|}{ Informed consent } & & $\mathrm{x}$ & & & & & & \\
\hline \multicolumn{2}{|c|}{ Randomization } & & $\mathrm{x}$ & & & & & & \\
\hline \multicolumn{2}{|c|}{ Allocation } & & $\mathrm{x}$ & & & & & & \\
\hline \multicolumn{10}{|c|}{ INTERVENTIONS: } \\
\hline \multicolumn{10}{|c|}{ Real Acupuncture } \\
\hline \multicolumn{10}{|c|}{ Sham Acupuncture } \\
\hline \multicolumn{10}{|c|}{ ASSESSMENTS: } \\
\hline \multicolumn{2}{|c|}{ UPSIT-TC } & $x$ & & & $\mathrm{x}$ & & & $\mathrm{x}$ & \\
\hline \multicolumn{2}{|r|}{ ASOF } & & $\mathrm{x}$ & & $\mathrm{x}$ & $\mathrm{x}$ & & $\mathrm{x}$ & $\mathrm{x}$ \\
\hline \multicolumn{2}{|c|}{ sQOD-NS } & & $\mathrm{x}$ & & $\mathrm{x}$ & $\mathrm{x}$ & & $\mathrm{x}$ & $\mathrm{x}$ \\
\hline
\end{tabular}

Figure 2

SPIRIT figure showing time points for enrolment, allocation, interventions, and assessment.

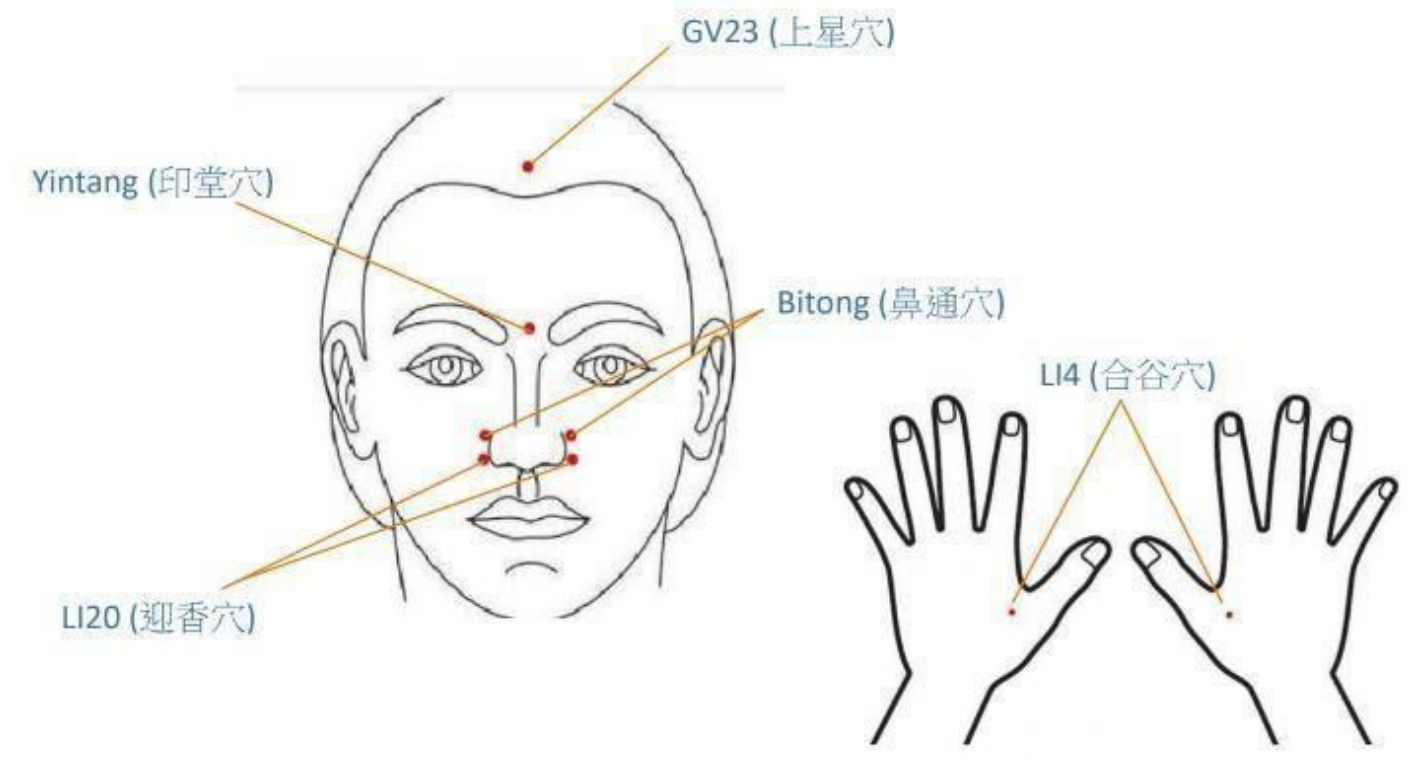

Figure 3

Locations of the acupuncture points

Supplementary Files 
This is a list of supplementary files associated with this preprint. Click to download.

- SupplementFiles1ScreeningSheetEng30062021.docx

- SupplementFiles2CRF30062021.docx

- SupplementFiles3ICFEng28022021.doc 